\title{
An Analysis of key process parameters for Hybrid Manufacturing by material extrusion and CNC machining
}

\author{
Rubén Paz \\ Universidad de Las Palmas de Gran Canaria, Departamento de Ingeniería Mecánica, Las Palmas de Gran \\ Canaria, 35017, Spain, phone: +34928459640, e-mail: ruben.paz@ulpgc.es
}

\author{
Javier Santamarta \\ Universidad de Las Palmas de Gran Canaria, Departamento de Ingeniería Mecánica, Las Palmas de Gran \\ Canaria, 35017, Spain, e-mail: javier.santamarta101@alu.ulpgc.es
}

\section{Mario D. Monzón}

Universidad de Las Palmas de Gran Canaria, Departamento de Ingeniería Mecánica, Las Palmas de Gran Canaria, 35017, Spain, phone: +34928458617 e-mail: mario.monzon@ulpgc.es

\section{Joshua García}

Universidad de Las Palmas de Gran Canaria, Departamento de Ingeniería Mecánica, Las Palmas de Gran Canaria, 35017, Spain, phone: +34928458603 e-mail: joshua.garcia@ulpgc.es

\section{Eujin Pei}

Brunel University London, Institute of Materials and Manufacturing, Kingston Lane, Uxbridge, UB8 3PH United Kingdom, phone: +44 (0)1895 268055, e-mail: eujin.pei@brunel.ac.uk

\begin{abstract}
Hybrid Manufacturing combines the advantages of Additive Manufacturing (AM) and Subtractive Manufacturing on a single machine. Although previous research has provided a good background of the manufacturing parameters, the analysis is often carried out separately and there is a lack of combined knowledge. The purpose of this research is to examine the influence of the main manufacturing parameters involved in AM and subtractive processes using different variables. Particularly, the study is focused on the Material Extrusion process concerning the layer height, fill angle and fill density; as well as two factors related to machining, including step-over and pass direction. The parameters that were examined include the dimension, hardness, flatness, weight and roughness. A multifactorial Design of Experiments (DoE) was proposed with a total of 64 samples. Next, a statistical analysis was carried out to assess the influence of the different groups on the response variables. Finally, a decision table presented and facilitated the selection of parameters depending on the desired objectives, leading to a framework that was applied to a case study for validation. This decision guide could enable designers and engineers to select the best strategy for a specific application, leading to a more efficient approach for manufacturing.
\end{abstract}

\section{Keywords}

Hybrid Manufacturing, Material Extrusion, Additive Manufacturing, Subtractive Manufacturing, Machining, Statistical Analysis 


\section{INTRODUCTION}

Hybrid Manufacturing (HM) consists of the integration of Additive and Subtractive manufacturing on a single machine. This idea aims to capitalise on the advantages of both technologies. In the case of Additive Manufacturing (AM), the main benefit is the capability to create complex geometries with minimal waste of material and reduced manufacturing times. On the other hand, Subtractive Manufacturing (SM) improves the quality of the finishing. While other researchers have experimented with the use of chemical methods such as the use of acetone to improve the surface finish quality, NCmachining offers better control [1,2]. Previous research has shown that HM allows material costs to be reduced up to $97 \%$, compared to traditional methods of SM [3]. HM machines have been developed since the 1990s [4], especially for metal fabrication. Recent work has also focused on hybrid laser metal deposition and considering critical factors such as processing temperatures, temperature gradients, and solidification conditions with regard to material properties such as lamellar interface cracking [5]. However, the rapid growth of material extrusion AM technologies (usually known as FDM) has led to a higher interest of HM methods for plastic components. The most common strategy is to create an AM part with the enough dimensions to be milled later, thus removing the extra surface material [6] to obtain the final desired dimensions. According to the literature [7], hybrid manufacturing has demonstrated an improved efficiency at microscale manufacturing compared to other post-processing methods.

Among others, one particular application is the biomedical devices. This sector has experimented important improvements in the last years due to the scaffolds fabrication by AM, especially caused by the increase of materials available for extrusion-based 3D printing. Moreover, the correct control of certain parameters in the FDM manufacturing process may result in high quality implants [8], but the surface finishing cannot be deeply controlled as it is inherent to the layer by layer fabrication. On the other hand, the porosity of the implant is a factor that directly affects the cell proliferation [9]. Therefore, the surface finishing is a key parameter in which hybrid manufacturing may suppose a substantial improvement for specific applications. A clear example of this is the hip prosthesis where some areas requires high porosity (for example coming from AM processes) and in other areas the good finishing (even at mirror level) is crucial for the correct functionality.

This work focuses on analyzing different manufacturing parameters related to material extrusion and machining. The main objective is to determine the influence of the build and machining factors to achieve different finishing qualities.

\section{METHODS}

\subsection{Manufacturing factors and parameters measured}

Although HM can occur within a single machine with Additive and Subtractive capabilities, this pilot study was carried out using a BQ Prusa i3 AM machine and a Mazak VTC 300 II CNC milling machine. Although the experiments may not have fully demonstrated the capabilities of a single machine, the fundamental manufacturing process is still close to identical to those being built from a single system. The manufacturing factors analysed in the case of the AM process were the layer height, fill 
angle and fill density. The layer height is a crucial parameter for material extrusion AM machines as it influences the quality of the external surface and manufacturing time. For example, a higher layer height could reduce manufacturing time but may influence the surface quality. The fill angle corresponds to the direction of the rectilinear internal fill related to the $\mathrm{X}$-axis of the AM machine. Finally, the fill density is the percentage that represents the amount of material used for the internal cavity whereby a $100 \%$ value would mean that the AM component would be completely solid. In the case of the subtractive process, the factors analysed were the stepover and pass direction. The stepover determines the amount of material that is machined in each lateral pass of the tool during the machining. This value is set up as a percentage of the tool diameter. Finally, the pass direction determines the angle of the tool during the machining related to the $X$ axis of the milling machine.

Five factors were analysed for two different levels following a multifactorial Design of Experiments (DoE) $\left(2^{5}=32\right.$ different groups). For each group, two samples were produced, leading to 64 samples. The samples were cubes of $15 \mathrm{~mm}$ side and the material used was PLA (1.75mm filament diameter). The printing parameters such as extrusion temperature, speeds, etc., were adjusted for PLA to ensure that the quality of prints was acceptable. According to the literature $[10,11]$, the extrusion temperature for PLA is usually between 205 and $220^{\circ} \mathrm{C}$, with deposition speed federate around $60 \mathrm{~mm} / \mathrm{min}$ and $50-60^{\circ} \mathrm{C}$ for the printing bed. Moreover, the extrusion multiplier must be close to 1 to avoid porosity [11], which in this application could result in better surface finishing. Starting from these recommended values, several 3D printing tests were performed to fit the final parameters until a good 3D printing quality was achieved. The final manufacturing parameters used were: $210^{\circ} \mathrm{C}$ of extrusion temperature $\left(220^{\circ} \mathrm{C}\right.$ for the first layer), 2 perimeters, 6 solid layers on the top and bottom of the samples, rectilinear infill pattern, 40 and $50 \mathrm{~mm} / \mathrm{s}$ of deposition speed (federate) for the perimeters and infill respectively, $0.6 \mathrm{~mm}$ extrusion width and 1 for the extrusion multiplier. The 3D printer did not have heated bed, so that the printing bed temperature was the room temperature. The response variables include aspects of dimensions, hardness, flatness, weight and roughness. Table 1 summarizes all the factors and parameters for this analysis.

Table 1. Factors-levels analysed and parameters measured.

\begin{tabular}{|l|l|l|}
\hline \multicolumn{2}{|l|}{ Factors } & \multirow{2}{*}{$\begin{array}{l}\text { Parameters } \\
\text { Measured }\end{array}$} \\
\hline Description & Levels & Dimensions \\
Layer height & 0.2 and $0.3 \mathrm{~mm}$ & Hardness \\
& 90 and $45^{\circ}$ & Flatness \\
Fill angle & 30 and $70 \%$ & Weight \\
Fill density & 50 and $60 \%$ of the tool diameter & Roughness \\
\hline Stepover & 90 and $45^{\circ}$ & \\
\hline Pass direction & &
\end{tabular}

\subsection{Experimental procedure}

According to the DoE, the manufacturing code for the 64 samples was generated using Slic3r software. Subsequently, the parts were fabricated and catalogued in a numbered sequence for analysis. Figure 1 shows the general workflow followed with 
the samples. For all the parameters assessed, three measurements were taken and the mean value was calculated.

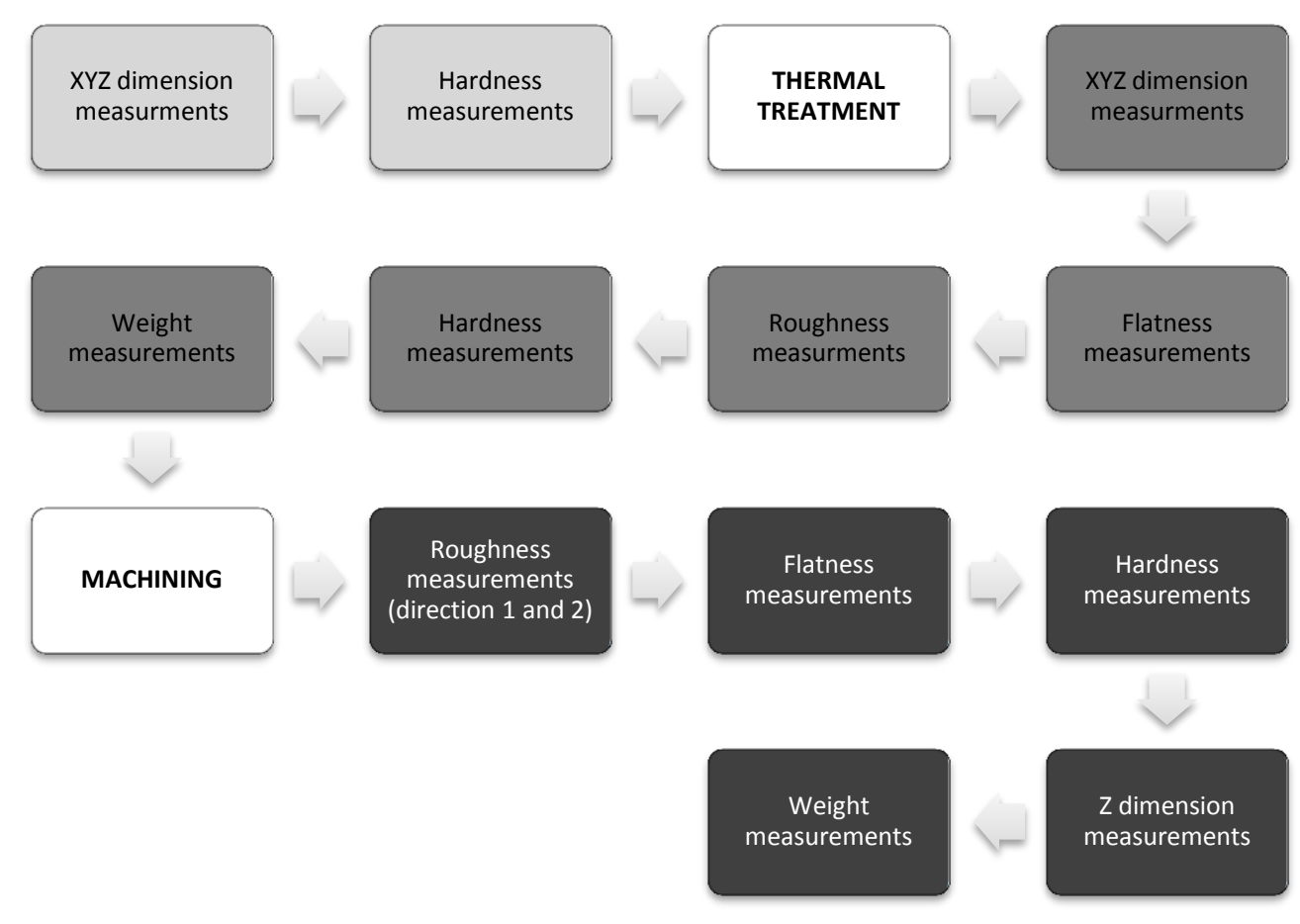

Figure 1. General workflow of the measurements taken.

Firstly, the XYZ dimensions and hardness (Shore D) were measured using a digital caliper and a hardness meter. Next, a thermal treatment was applied by heating the samples at $63^{\circ} \mathrm{C}$ for 5 hours and then cooling the samples down for 48 hours to room temperature in a hermetic container with silica salt. The purpose of the thermal treatment is to exceed the glass transition temperature of the material to modify the crystallinity of the part and improve the physical properties.

In parallel to the general workflow and with the aim to assess the impact of the thermal treatment in the mechanical properties, several flexural samples were produced using the same AM machine and divided into two different groups. One of the groups was thermally treated while the other group was not. Finally, the samples were tested under flexural load in accordance to the guidelines from ISO 178:2001 standard (Plastics. Determination of flexural properties).

Once the thermal treatment was applied, the XYZ dimensions were measured once again, as well as for parameters including flatness, roughness and hardness.

The next important step was to machine the samples. Autodesk Fusion 360 Computer Aided Machining (CAM) software was used to generate sets of toolpaths having different combinations of stepovers and pass directions according to the DoE. A 
parallel operation was defined in the software, with a $0.6 \mathrm{~mm}$ stepdown (a $14.6 \mathrm{~mm}$ theoretical height). A $5 \mathrm{~mm}$ flat endmill tool was used. For the experiments, a specially built clamp was designed, built and installed to securely fasten the samples in the milling machine. The machining speeds and cooling system were adjusted in accordance to the machining guidelines. The surface speed of the tool was set in $5.445 \mathrm{~m} / \mathrm{min}$ and the feed per tooth was set to $0.075 \mathrm{~mm} /$ tooth. Compressed air was applied to cool the machining process to ensure that plastic components do not become overheated, therefore semi-melted. After the machining, the roughness, flatness and hardness were systematically recorded. For consistency, the roughness level was measured in two perpendicular directions and the Z-height was also recorded.

\subsection{Statistical Analysis}

Once all the measurements were taken, a statistical analysis was applied to assess the influence of the manufacturing factors in the parameters used. Three different analyses were carried out. The first one was accomplished taking into account the three factors of the AM process (layer height, fill angle and fill density). The second analysis was carried out taking into account the two factors of the machining process (stepover and pass direction) and the measurements after the machining. For both analyses, the procedure followed was the depicted in Figure 2. However, as the number of factors was not equal ( 3 factors for $A M$ and 2 factors for SM), a 2-way and n-way ANOVA were applied respectively. These analysis could be useful to select the best combinations of manufacturing factors for AM and SM, but this work will focus on the hybrid manufacturing process. A third statistical analysis was developed using the 5 factors studied (layer height, fill angle and fill density for AM; and stepover and pass direction for SM) and the parameters measured after machining. For each parameter, the data were organized according to the 32 different groups of the DoE. For each group, an initial analysis was carried out to assess whether the data followed a normal distribution. If the data did not follow a normal distribution, a Kruskal Wallis test would then be applied to determine if any group would be significantly different to the rest (99\% significance level). If this occurs, then a multi-comparison test would be applied to identify the groups that show a significant difference (95\% significance level). If the data show a normal distribution, then a one-way ANOVA is applied to assess if there is any group that shows a significant difference ( $95 \%$ significance level). Likewise, a final multi-comparison test was applied to identify which groups were significantly different. Figure 2 summarizes the statistical workflow. 


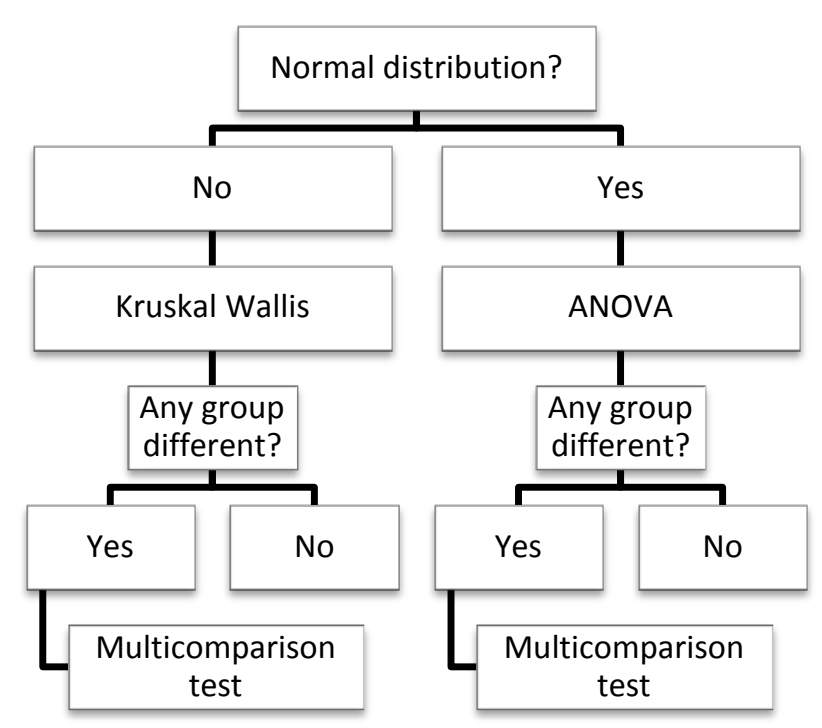

Figure 2. Statistical analysis workflow.

\section{RESULTS}

\subsection{Thermal treatment}

From the thermal treatment and flexural tests carried out, the results showed that the treated samples obtained an elastic flexural modulus $2.5 \%$ higher than the non-treated samples, which meant no significant differences were present. It was observed that there was a clear increase of the hardness (Figure 3 ) and a significant distortion on the $\mathrm{XYZ}$ dimensions. Since the thermal treatment is more effective on the surface rather than in the interior areas of the samples, this increased the hardness of the samples. This improvement on the surface is probably due to the increase of degree of crystallization of PLA. However, after the machining, the hardness decreased slightly as the most external layers were removed.

\section{Hardness (Shore D)}

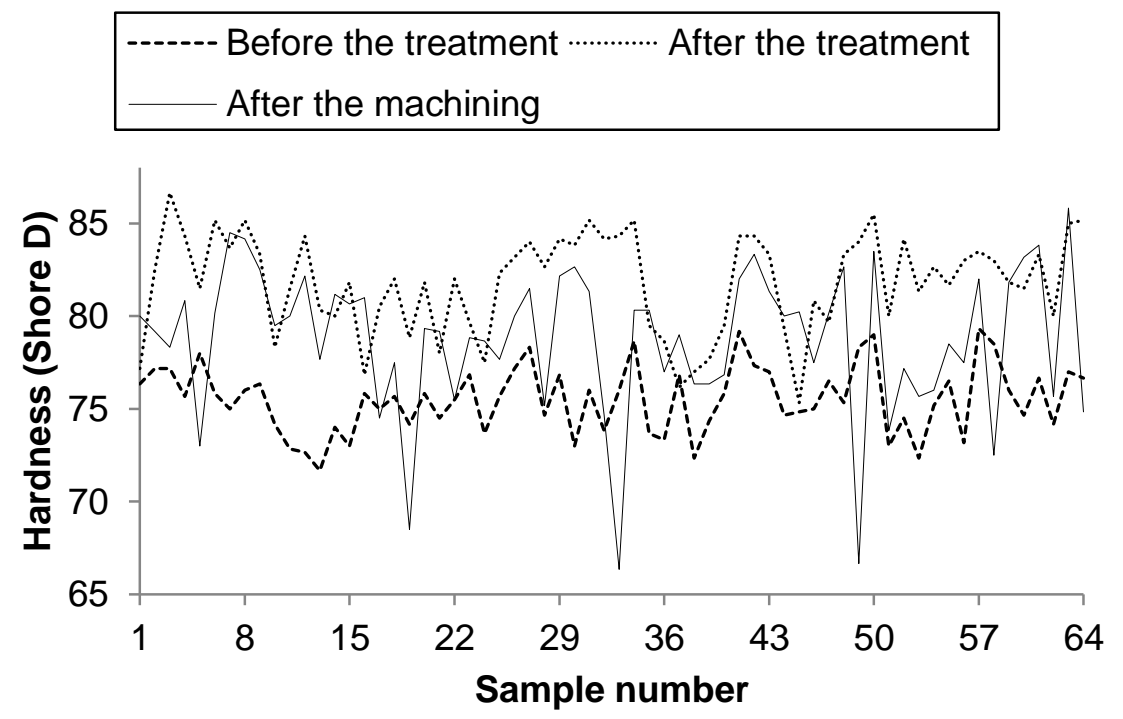

Figure 3. Graph of the hardness of the 64 samples before the treatment. 


\subsection{Comparison before and after the machining}

The machining process also enabled an improvement of finishing parameters such as flatness and roughness. Figure 4 shows the flatness measured before and after the machining for each one of the samples. Figure 5 shows the roughness measured before and after the machining for each sample. Both results show that the machining process improved the flatness and roughness qualities.

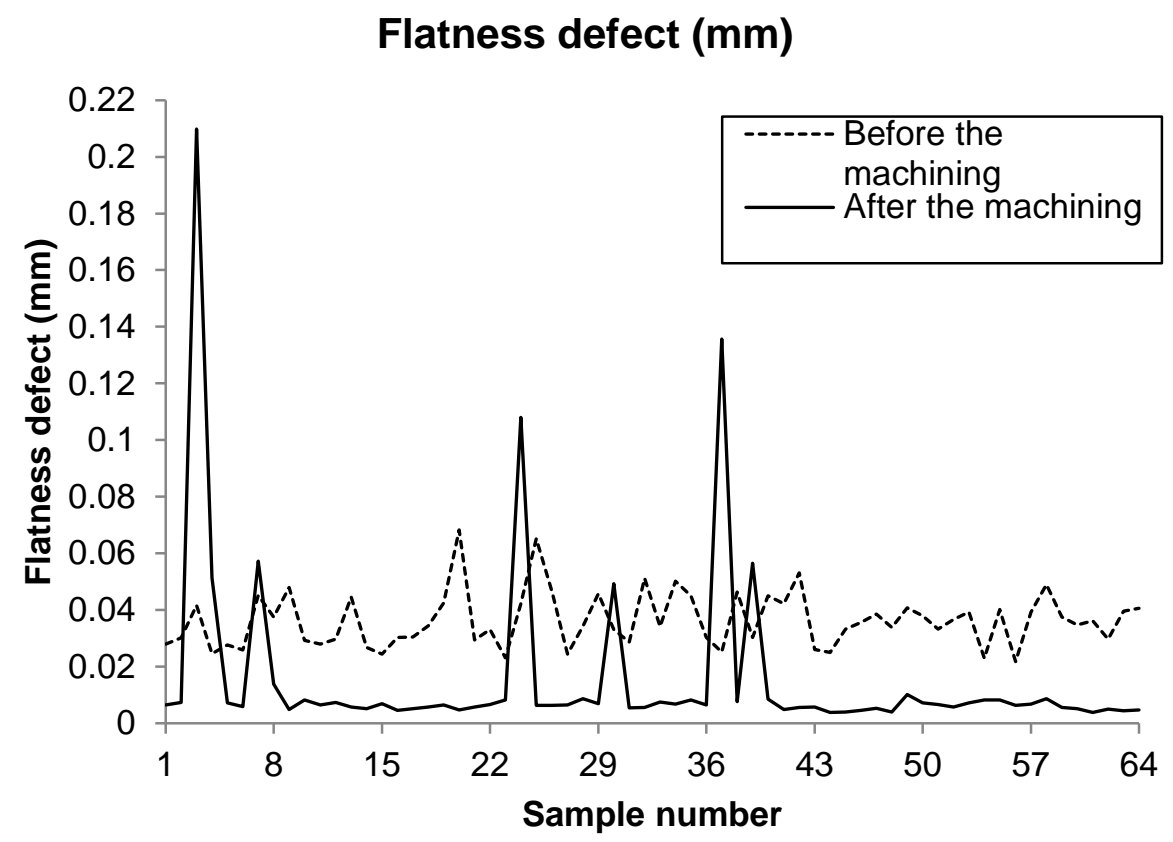

Figure 4. Graph of the flatness defect of the 64 samples before and after the machining.

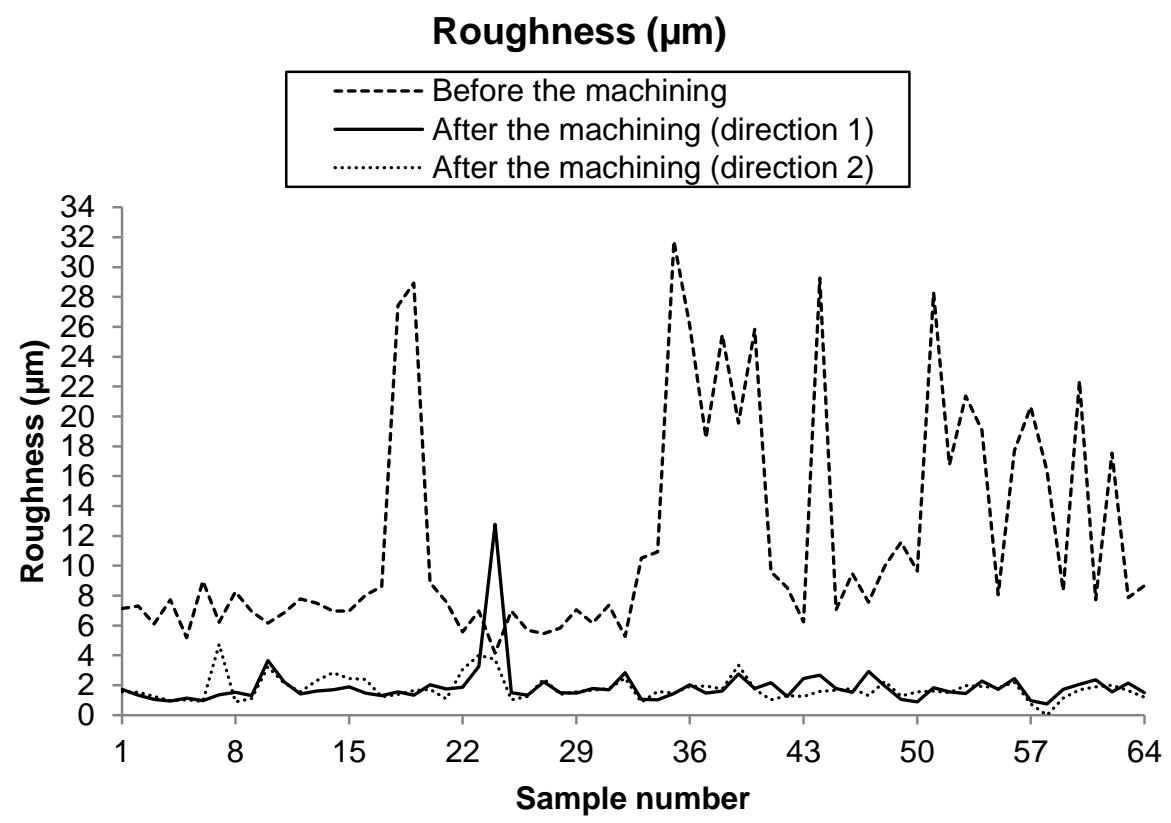


Figure 5. Graph of the roughness of the 64 samples before and after the machining.

\subsection{Results of the statistical analysis}

According to the statistical analysis, significant differences were found for the roughness and $Z$ dimension. In the case of roughness, groups 29 and 12 were statistically different. In the case of the $Z$ dimension, group 29 was significantly different from groups 1, 2, 4, 5, 6, 10 12, 14, 15, 18, 19 and 20. On the other hand, it was also observed that the statistical analysis could not find significant differences in some cases due to the low number of samples. When the number of samples is low, the multi-comparison test requires significant differences to reject the null hypothesis and thus conclude that those groups are different. For this reason, a subsequent analysis was developed to create a decision table.

\section{VALIDATION OF DECISION TABLE}

To create a decision table to select the best manufacturing factors, the graphs from the multi-comparison tests were integrated in order to enable a pre-selection of the best groups of each parameter. For example, for hardness (Figure 6), groups 4, 15 and 21 were selected in that order since they were the groups with the highest hardness results. This procedure was applied for each parameter measured, cumulating in a table that allows the selection of the best 1-3 groups for each parameter. Table 2 shows this table that has been filtered to show the best 2 possible groups of each parameter. Note that the ideal group should minimize the roughness, flatness and weight and maximize the hardness and the accuracy on the $Z$ dimension (compared with the theoretical value, which is $14.4 \mathrm{~mm}$ ). 


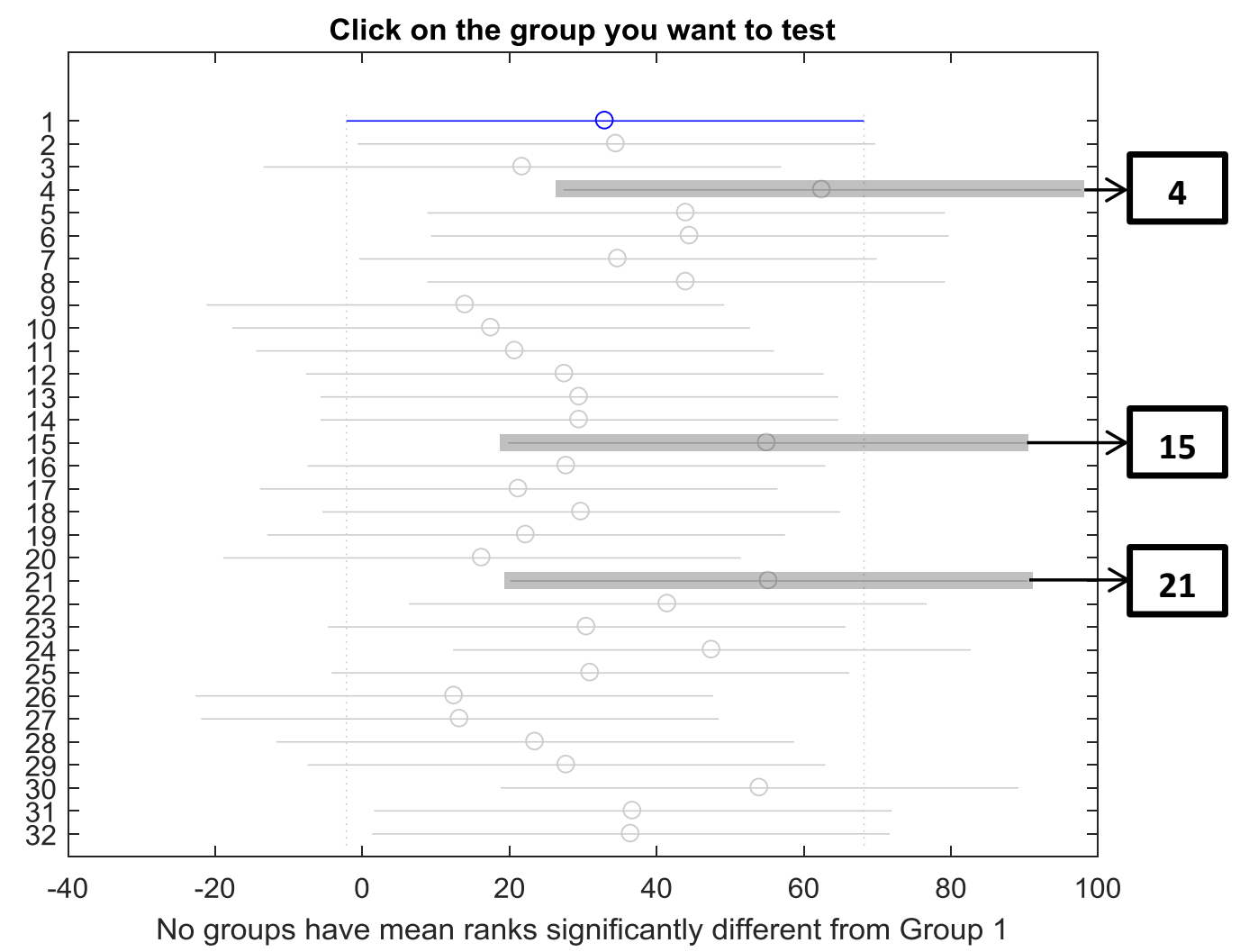

Figure 6. Multi-comparison graph for hardness and the selection of the best groups.

Table 2. Decision table filtered with the best 2 groups for each parameter measured.

\begin{tabular}{|c|c|c|c|c|c|c|c|}
\hline $\begin{array}{l}\text { Parameter } \\
\text { measured }\end{array}$ & $\begin{array}{l}\text { Best } \\
\text { groups }\end{array}$ & Ranking & $\begin{array}{l}\text { Layer height } \\
(\mathrm{mm})\end{array}$ & $\begin{array}{c}\text { Fill } \\
\text { angle }\left({ }^{\circ}\right)\end{array}$ & $\begin{array}{l}\text { Fill density } \\
\text { (\%) }\end{array}$ & $\begin{array}{c}\text { Stepover (\% } \\
\varnothing)\end{array}$ & $\begin{array}{c}\text { Pass } \\
\text { direction }\left({ }^{\circ}\right)\end{array}$ \\
\hline \multirow{2}{*}{$\begin{array}{l}\text { Roughness } \\
\text { (direction 1) }\end{array}$} & 29 & 1 & 0.3 & 45 & 70 & 50 & 90 \\
\hline & 2 & 2 & 0.2 & 90 & 30 & 60 & 90 \\
\hline \multirow{2}{*}{$\begin{array}{c}\text { Roughness } \\
\text { (direction 2) }\end{array}$} & 29 & 1 & 0.3 & 45 & 70 & 50 & 90 \\
\hline & 3 & 2 & 0.2 & 90 & 30 & 50 & 45 \\
\hline \multirow{2}{*}{ Flatness } & 23 & 1 & 0.3 & 90 & 70 & 50 & 45 \\
\hline & 31 & 2 & 0.3 & 45 & 70 & 50 & 45 \\
\hline \multirow{3}{*}{ Hardness } & 4 & 1 & 0.2 & 90 & 30 & 60 & 45 \\
\hline & 15 & 1 & 0.2 & 45 & 70 & 50 & 45 \\
\hline & 16 & 2 & 0.2 & 45 & 70 & 60 & 45 \\
\hline \multirow{3}{*}{$\begin{array}{c}\text { Z Dimension } \\
\text { (accuracy to } \\
14.4 \mathrm{~mm} \text { ) } \\
\end{array}$} & 13 & 1 & 0.2 & 45 & 70 & 50 & 90 \\
\hline & 9 & 2 & 0.2 & 45 & 30 & 50 & 90 \\
\hline & 25 & 2 & 0.3 & 45 & 30 & 50 & 90 \\
\hline \multirow{2}{*}{ Weight } & 20 & 1 & 0.3 & 90 & 30 & 60 & 45 \\
\hline & 19 & 2 & 0.3 & 90 & 30 & 50 & 45 \\
\hline
\end{tabular}

According to the decision table, thinner layers can lead to more hardness and accuracy. However, the lowest weight is achieved with high values of layer height. As expected, the lightest samples are also obtained with the lowest values of fill density. In terms of flatness, the best values were achieved with high values of fill density (a 
stiffer structure leads to a better flatness even after the machining). Regarding the roughness, there is not a clear influence of only one parameter, but it is clear that the parameters of group 29 achieve the best results in both directions.

Although this decision table allows users to select the best groups for each parameter, in some cases the objective may not be limited to just one parameter, but several. For such cases, the procedure would require ranking the groups for each parameter according to the average value of the samples. This process is explained in the following case study.

\subsection{Case Study}

The case study utilized the design of a camera housing in which the external profile (Figure 7) would be produced using vacuum casting. A silicone mold would be manufactured, which it also implies the production of the model of the camera. To obtain this model, hybrid manufacturing will be used to take advantage of AM processes to achieve a high fidelity prototype combined with good finishing qualities of machining.
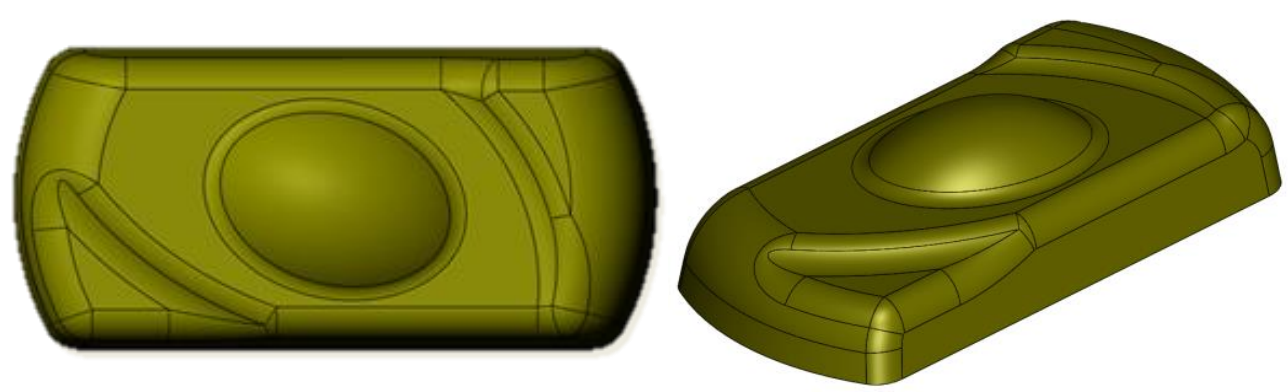

Figure 7. Final desired geometry for the camera model.

In this case, the objective was to obtain a low roughness, low flatness defect and low weight yet having high hardness and high accuracy on the $Z$ dimension. Since Table 2 is not enough to take a decision among all the preselected groups, a ranking sequence was determined for each parameter. To do so, only the best two groups were filtered for each parameter. Subsequently, the rankings were calculated depending on the average value of each parameter. Finally, the sum of the ranking for each parameter measured was calculated so that the one with the lowest value will be the best one taking into account all the parameters. As the roughness was measured in two different directions, the average rank of both was used. Table 3 shows the details of this procedure. According to this, the best groups were groups 9 and 13. Finally, the manufacturing factors of group 9 were selected (Table $2,0.2 \mathrm{~mm}$ layer height, $45^{\circ}$ fill angle, $30 \%$ fill density, $50 \%$ stepover and $90^{\circ}$ pass direction).

Table 3. Final ranking of the best 2 groups of each parameter.

\begin{tabular}{|c|c|c|c|c|c|c|}
\hline \multirow[b]{2}{*}{ Group } & \multicolumn{5}{|c|}{ Ranking } & \multirow[b]{2}{*}{$\begin{array}{l}\text { Final } \\
\text { ranking }\end{array}$} \\
\hline & Roughness & Flatness & Hardness & $\begin{array}{l}\text { Z Dimension } \\
\text { (accuracy } \\
\text { compared }\end{array}$ & Weight & \\
\hline
\end{tabular}




\begin{tabular}{|c|c|c|c|c|c|c|c|}
\cline { 1 - 3 } & Dir. 1 & Dir. 2 & & & to 14.4mm) & & \\
\hline 9 & 6 & 5 & 3 & 12 & 2 & 3 & 25.5 \\
\hline 13 & 5 & 4 & 5 & 6 & 1 & 9 & 25.5 \\
\hline 3 & 4 & 2 & 6 & 10 & 4 & 5 & 28 \\
\hline 23 & 10 & 8 & 1 & 5 & 5 & 8 & 28 \\
\hline 25 & 2 & 6 & 8 & 13 & 3 & 4 & 32 \\
\hline 31 & 11 & 10 & 2 & 3 & 6 & 11 & 32.5 \\
\hline 2 & 3 & 3 & 13 & 4 & 9 & 6 & 35 \\
\hline 29 & 1 & 1 & 7 & 9 & 11 & 10 & 38 \\
\hline 15 & 9 & 7 & 9 & 2 & 8 & 12 & 39 \\
\hline 4 & 7 & 13 & 11 & 1 & 13 & 7 & 42 \\
\hline 19 & 8 & 9 & 12 & 8 & 12 & 2 & 42.5 \\
\hline 16 & 13 & 11 & 4 & 7 & 7 & 13 & 43 \\
\hline 20 & 12 & 12 & 10 & 11 & 10 & 1 & 44 \\
\hline
\end{tabular}

Once the manufacturing parameters were selected, the CAD geometry of the camera was prepared by adding $0.6 \mathrm{~mm}$ extra thickness to the surface (except the bottom) to be later machined. A total of nine layers and perimeters were established for the top and surface features of the part to guarantee that there would be at least $1.8 \mathrm{~mm}$ of solid thickness before machining. Apart from this, a $10 \mathrm{~mm}$ cube was included at the side of the model to use the center of the top face as the work offset for machining. In addition, three holes were added to the bottom. The part was produced using AM taking a total of 5 hours and then secured onto the machine bed (Figure 8).

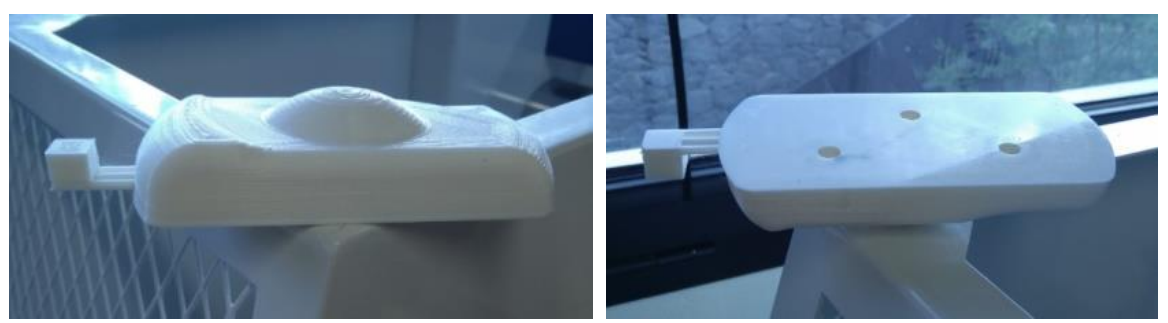

Figure 8. 3D printed camera.

Autodesk Fusion 360 was used to generate the machining code and the work offset was defined at the center of the top face of the cube. Four operations were created: a contour operation with a $16 \mathrm{~mm}$ flat endmill tool to machine the bottom contour, a horizontal operation with a $6 \mathrm{~mm}$ flat endmill tool to machine the top horizontal faces, and a contour and parallel operations with a $6 \mathrm{~mm}$ ball endmill tool to machine the final surface. The surface speed and feed per tooth were defined by increasing three times the values depicted in section 2.2. Moreover, these values were multiplied by the tool diameter ratio compared to the $5 \mathrm{~mm}$ tool used in the samples to avoid excessive heating of the PLA material during machining. Compressed air was used for cooling the part and the overall process of machining took 20 minutes to complete (Figure 9). 

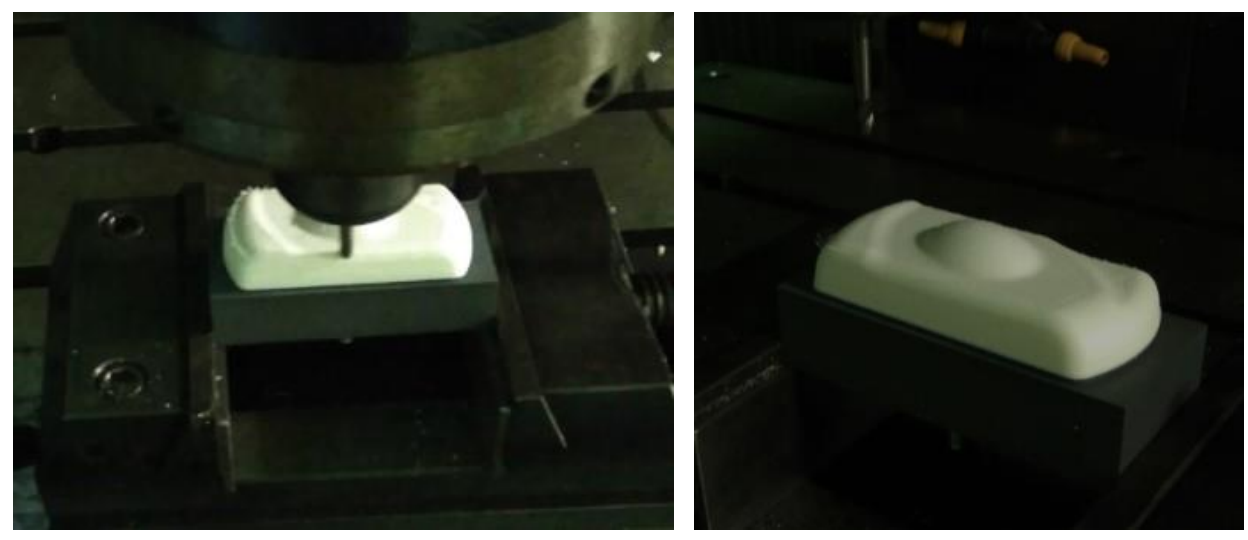

Figure 9. Camera during the machining (left) and part obtained (right)

The part obtained showed high finishing qualities, although it was observed that in some areas, the filaments of the solid top layer were ripped out instead of machined. This may be due to bad adhesion between the interior filaments of the first solid layer and the filaments of the perimeters and fill structure. In Figure 10, it can be observed that this was only limited to some perimeter areas of the top layers.

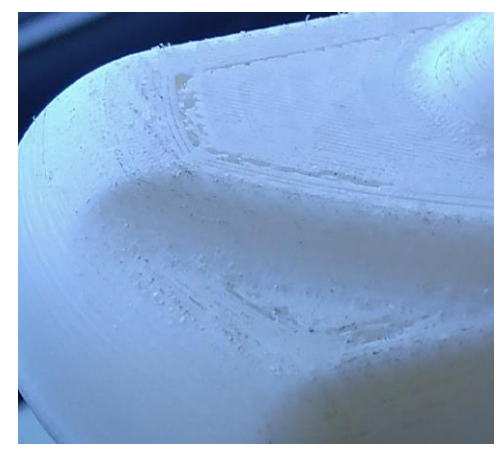

Figure 10. Defects caused potentially by a poor adhesion between filaments

\section{CONCLUSIONS}

The influence of layer height, fill angle, fill density, stepover and pass direction on finishing parameters such as dimensions, hardness, flatness, weight and roughness, was analysed. According to statistical analysis, significant differences were found for roughness and $Z$ dimension. For other parameters, no significant differences were found. From the graphs of the multi-comparison tests, a selection of the best groups for each parameter was carried out and shown in Table 2 that allows for the selection of the best manufacturing factors depending on the parameter that a user wishes to achieve. According to these results, thinner layers can lead to more hardness and accuracy, while high values of layer height result in reduced weight. The lightest samples are also obtained with the lowest values of fill density, while the highest fill densities achieve better flatness (stiffer structure). Regarding the roughness, there is not a clear influence of only one parameter, but group 29 achieves the best results in both directions.

If the application requires the optimization of several parameters, then the procedure using the decision table would require sorting of the groups with the average values of 
the samples. The total ranking score is also calculated so that the lowest value will be the best manufacturing strategy for the specific application. A comparison of the samples showed that machining improved the finishing parameters such as roughness and flatness defect. The process of thermal treatment also enhances the hardness of the parts. Regarding the elastic flexural modulus, the thermal treatment achieves similar results to non-treated samples, with a slight improvement of $2.5 \%$. Although the findings of this work is limited to a small number of samples and with a base study to validate the claims, it extends existing knowledge by providing a clear correlation of the AM and SM parameters in a single decision table. This decision guide could enable designers and engineers to select the best strategy for a specific application, leading to a more efficient approach for manufacturing. It is hoped that future work will extend other HM parameters to be included as well as to ascertain the findings using different production materials.

\section{REFERENCES}

1. Chohan, JS, Singh, R (2017) Pre and post processing techniques to improve surface characteristics of FDM parts: a state of art review and future applications, Rapid Prototyping Journal, 23(3): 495-513

2. Maidin, S., Muhamad, M. K., Pei, E. (2015) Experimental Setup for UltrasonicAssisted Desktop Fused Deposition Modeling System, Applied Mechanics \& Materials, 761,: 324-328

3. Yamazaki, T. (2016) Development of A Hybrid Multi-Tasking Machine Tool: Integration of Additive Manufacturing Technology with CNC Machining, Procedia CIRP, 42: 81-86

4. Lorenz, KA, Jones, JB, Wimpenny, DI, Jackson, MR (2015), A review of hybrid manufacturing. Solid Freeform Fabrication Symposium (SFF), Austin, TX, Aug, 10-12

5. Seidel, A. et al. (2018) Added value by hybrid additive manufacturing and advanced manufacturing approaches, Journal of Laser Applications 30: 032301

6. Du, W, Qian B, y Bi Z (2016), A Novel Method for Additive/Subtractive Hybrid Manufacturing of Metallic Parts, Procedia Manufacturing, 5: 1018-30

7. Chu, Won-Shi, Chung-Soo Kim, Hyun-Taek Lee, Jung-Oh Choi, Jae-Il Park, Ji-Hyeon Song, Ki-Hwan Jang, y Sung-Hoon Ahn. 2014. Hybrid Manufacturing in Micro/Nano Scale: A Review. International Journal of Precision Engineering and ManufacturingGreen Technology, 1 (1): 75-92

8. Prashanth, R., Panos SS, Avinash, DT (2017), Analyzing the Effects of Temperature, Nozzle-Bed Distance, and Their Interactions on the Width of Fused Deposition Modeled Struts Using Statistical Techniques Toward Precision Scaffold Fabrication, Journal of Manufacturing Science and Engineering, 139(7): 071007-1-071007-9 
9. Nowicki, M, Castro, N, Plesniak, MW, Zhang, LG (2016) 3D printing of novel osteochondral scaffolds with graded microstructure, Nanotechnology ,27 (41): 414001

10. A. Lanzotti, M. Grasso, G. Staiano, y M. Martorelli, The impact of process parameters on mechanical properties of parts fabricated in PLA with an open-source 3D printer, Rapid Prototyping Journal, vol. 21, n. ${ }^{\circ}$ 5, pp. 604-617, ago. 2015.

11. E. G. Gordeev, A. S. Galushko, y V. P. Ananikov, Improvement of quality of 3D printed objects by elimination of microscopic structural defects in fused deposition modeling, PLOS ONE, vol. 13, n.o 6, p. e0198370, jun. 2018. 